\title{
Functional genomics of fuzzless-lintless mutant of Gossypium hirsutum L. cv. MCU5 reveal key genes and pathways involved in cotton fibre initiation and elongation
}

Kethireddy Venkata Padmalatha', Deepak P Patil ${ }^{2}$, Krishan Kumar², Gurusamy Dhandapani', Mogilicherla Kanakachari ${ }^{1}$, Mullapudi LV Phanindra', Saravanan Kumarr ${ }^{2}$ T C Mohan ${ }^{3}$, Neha Jain ${ }^{1}$, Arkalgud H Prakash ${ }^{4}$, Hiremath Vamadevaiah ${ }^{3}$, Ishwarappa S Katageri ${ }^{3}$, Sadhu Leelavathi ${ }^{2}$, Malireddy K Reddy ${ }^{2}$, Polumetla Ananda Kumar $^{1}$ and Vanga Siva Reddy ${ }^{2^{*}}$

\begin{abstract}
Background: Fuzzless-lintless cotton mutants are considered to be the ideal material to understand the molecular mechanisms involved in fibre cell development. Although there are few reports on transcriptome and proteome analyses in cotton at fibre initiation and elongation stages, there is no comprehensive comparative transcriptome analysis of fibre-bearing and fuzzless-lintless cotton ovules covering fibre initiation to secondary cell wall (SCW) synthesis stages. In the present study, a comparative transcriptome analysis was carried out using G. hirsutum L. Cv. MCU5 wild-type (WT) and it's near isogenic fuzzless-lintless (f) mutant at fibre initiation ( $0 \mathrm{dpa} /$ days post anthesis), elongation (5, 10 and $15 \mathrm{dpa}$ ) and SCW synthesis (20 dpa) stages.

Results: Scanning electron microscopy study revealed the delay in the initiation of fibre cells and lack of any further development after $2 \mathrm{dpa}$ in the $\mathrm{fl}$ mutant. Transcriptome analysis showed major down regulation of transcripts (90\%) at fibre initiation and early elongation (5 dpa) stages in the $\mathrm{fl}$ mutant. Majority of the down regulated transcripts at fibre initiation stage in the $\mathrm{fl}$ mutant represent calcium and phytohormone mediated signal transduction pathways, biosynthesis of auxin and ethylene and stress responsive transcription factors (TFs). Further, transcripts involved in carbohydrate and lipid metabolisms, mitochondrial electron transport system (mETS) and cell wall loosening and elongation were highly down-regulated at fibre elongation stage (5-15 dpa) in the $\mathrm{fl}$ mutant. In addition, cellulose synthases and sucrose synthase C were down-regulated at SCW biosynthesis stage (15-20 dpa). Interestingly, some of the transcripts ( 50\%) involved in phytohormone signalling and stress responsive transcription factors that were up-regulated at fibre initiation stage in the WT were found to be up-regulated at much later stage (15 dpa) in $\mathrm{fl}$ mutant.

Conclusions: Comparative transcriptome analysis of WT and its near isogenic $\mathrm{fl}$ mutant revealed key genes and pathways involved at various stages of fibre development. Our data implicated the significant role of mitochondria mediated energy metabolism during fibre elongation process. The delayed expression of genes involved in phytohormone signalling and stress responsive TFs in the $\mathrm{fl}$ mutant suggests the need for a coordinated expression of regulatory mechanisms in fibre cell initiation and differentiation.
\end{abstract}

\footnotetext{
* Correspondence: vsreddy@icgeb.res.in

${ }^{2}$ Plant Transformation Group, International Center for Genetic Engineering and Biotechnology, Aruna Asaf Ali Marg, New Delhi 110067, India Full list of author information is available at the end of the article
} 


\section{Background}

Cotton is a commercially important fibre crop and is used as a major source of natural textile fibre and cottonseed oil. Among the four cultivated species, Gossypium hirsutum represents over $95 \%$ of the cultivated cotton worldwide whereas the other three species, $G$. barbadense, G. arboreum and G. herbaceum together represent the remaining 5\%. Cotton fibres are singlecelled seed trichomes that develop from the ovule epidermal cells. About $30 \%$ of the seed epidermal cells differentiate into spinnable fibres [1,2]. Cotton fibre development includes four distinct, but overlapping stages: initiation, elongation/primary cell wall (PCW) synthesis, secondary cell wall (SCW) synthesis and maturation. The fibre cell initiation usually occurs from 2-3 days before anthesis to $2-3$ days post anthesis (dpa) and fibre cell elongation occurs up to $20 \mathrm{dpa}$. However, fast elongation of fibre cell occurs between 5 to $15 \mathrm{dpa}$. Secondary cell wall synthesis starts at about $20 \mathrm{dpa}$ and continues up to $45 \mathrm{dpa}$. During this period large amount of cellulose (>90\%) deposition takes place and the fibre cell wall becomes thick. In the final maturation stage (45-50 dpa) fibres undergo dehydration and produce mature cotton lint [1-3].

Cotton fibre is considered as an excellent single-celled model system for studying the molecular mechanisms controlling the plant cell initiation, elongation and secondary cell wall biosynthesis. In recent years, functional genomics-based approaches have been widely used to investigate the genes involved in cotton fibre development [2-8]. Phytohormones such as ethylene [9], auxins $[10,11]$ and brassinosteroids (BR) $[12,13]$ and transcription factors such as MYB25 [14] and MYB25-like [15,16] were shown to be involved in fibre development. Cotton fibre elongates by diffusion growth mechanism and the turgor driven force is required for unidirectional elongation $[17,18]$. Therefore, osmotically active solutes (soluble sugars, potassium and malate) and ion-transporters $\left(\mathrm{H}^{+}\right.$-ATPases and $\mathrm{K}^{+}$-transporter) play an important role in maintaining the osmotic potential of the elongating fibre cell [18]. It is reported that the closure of plasmodesmata (PD) and the coordinated up-regulation of potassium $\left(\mathrm{K}^{+}\right)$and sugar transporters during fibre elongation stage maintains the turgor pressure required for the fibre cell elongation and the duration of PD closure correlates positively with the fibre length [19]. In addition, it has been reported that ROS (reactive oxygen species) homeostasis is the central regulatory mechanism for cotton fibre initiation and differentiation [8]. Carbohydrate and energy metabolisms play an important role in the fibre development by providing the carbon skeletons for the synthesis of cell wall polysaccharides and fatty acids $[5,7,20,21]$. Several studies have shown the role of xyloglucan and pectin modifying enzymes
[22], arabinogalactans [23] and expansins [24] in cell wall loosening and expansion during fibre elongation stage. Further actin cytoskeleton plays an important role during fibre elongation stage and reorientation of cytoskeleton microtubules is required for the onset of secondary cell wall synthesis [25-27]. Despite extensive research on cotton fibre biology over the last few decades, the mechanisms controlling fibre development remain largely unknown.

The fuzzless-lintless $(f l)$ ovules of cotton mutant are ideal material for identifying genes involved in the fibre development through comparative approaches. A few comparative transcriptome and proteome studies were carried out to understand the genes involved in fibre initiation and differentiation using the ovules of fuzzlesslintless mutant and its wild-type [4,8,28]. Similarly, a comparative proteome study was carried out using ovules of $f l$ mutant and its wild-type to identify the differentially expressed proteins at elongation stage [7]. However, comprehensive transcriptome studies employing fuzzless-lintless mutants involving all the important stages (initiation, elongation and secondary cell wall synthesis) of fibre development are not yet reported.

In the present study, comparative transcriptome analysis of $f l$ mutant with its wild-type (WT), G. hirsutum L. cv. MCU5 at fibre initiation (0 dpa), elongation (5, 10 and $15 \mathrm{dpa}$ ) and SCW synthesis stage (20 dpa) was carried out using Affymetrix cotton GeneChip genome array. Data from this study suggests that stress responsive transcription factors and the genes involved in calcium $\left(\mathrm{Ca}^{2+}\right)$ and phytohormone-mediated signalling pathways play a crucial regulatory role in fibre cell initiation and differentiation. Our study also revealed the down-regulation of several genes involved in interconversion of sugar molecules and mitochondrial electron transport system (mETS) that are required for the synthesis of cell wall polysaccharides and fatty acids and maintenance of redox-homeostasis during fibre elongation. The comprehensive transcriptome analysis identified several stage specific genes and pathways operating during the fibre development that might be useful for the improvement of cotton fibre.

\section{Results and discussion}

\section{Morphology of fuzzless-lintless mutant}

The near isogenic $f l$ mutant employed in this study is a spontaneous mutant of G. hirsutum L. cv. MCU5 first identified in 1984 [29] and maintained as a pure fuzzless-lintless line in the germplasm collections at the Cotton Breeding Station, Tamil Nadu Agricultural University, Coimbatore [29,30]. Morphological and growth parameters of the $f l$ mutant including plant height, leaf size, flower colour, number of bolls per plant, number of seeds per boll, etc. are very similar to that of WT. 
Scanning electron microscopy (SEM) study was carried out to identify the differences in early stages of fibre development by comparing the ovule of $f l$ mutant with that of WT (Figure 1A). SEM analysis revealed the presence of fibre cell initials in the $f l$ mutant, though very less in numbers as compared to WT. Fibre initials could be seen two days before anthesis ( $-2 \mathrm{dpa})$ in the WT and were more prominent at $0 \mathrm{dpa}$. Similarly, the fibre initials were also observed on the $f l$ mutant, though not very prominent at $-2 \mathrm{dpa}$, but were more visible at $0 \mathrm{dpa}$ (Figure 1A). This suggests the probable delay of fibre initiation in the $f l$ mutant. However, further growth of fibre initials were not observed in the $f l$ mutant leading to its fuzzless-lintless status (Figure 1A and B).

\section{Comparative transcriptome analysis}

Comparative transcriptome analysis was carried out at fibre initiation (0 dpa), elongation (5, 10 and $15 \mathrm{dpa}$ ) and SCW synthesis $(20 \mathrm{dpa})$ stages using ovules of $f l$ mutant and fibre-bearing ovules of WT to decipher molecular mechanisms involved in fibre cell development. Affymetrix cotton GeneChip genome arrays were used for transcriptome analysis. Transcripts with false discovery rate (FDR) adjusted $p$ value $\leq 0.01$ and fold change of $\geq 3.0$ were considered as differentially expressed transcripts (DETs) in the $f l$ mutant as compared to their respective stages in WT (Figure 1C, Additional file 1). The number of down-regulated transcripts was more as compared to up-regulated transcripts in various stages analysed. Further, the percentage of down-regulated transcripts was very high at fibre initiation (0 dpa, 90.0\%) and early elongation (5 dpa, 91.5\%) stages as compared to the later stages (Figure 1C). Data analysis revealed that majority of the DETs showed stage specific expression pattern with minor overlap among the stages (Figure 1D).

\section{Annotation and functional classification of DETs}

The DETs were annotated based on TAIR proteome database (http://www.arabidopsis.org). Out of 3,898 DETs, 2,968 (76.14\%) were matched with Arabidopsis gene models with $E$ value $\leq \mathrm{e}^{-10}$ (Additional file 1). Further, the DETs were classified into different functional categories according to their putative functions based on MIPS functional catalogue (http://mips.gsf.de/projects/ funcat). In categories such as "protein with binding function or cofactor requirement", "response to biotic and abiotic stresses", "transcription", "cellular transport, transport facilities and transport routes" and "biogenesis of cellular components" the transcripts were mostly down-regulated at fibre initiation and early elongation stages (Additional file 2). Further, DETs related to various transcription factor (TF) families and phytohormone biosynthesis and signal transduction pathways were identified using Arabidopsis transcription factor (http://plntfdb.bio.uni-potsdam.de) and hormone (http:// ahd.cbi.pku.edu.cn) databases, respectively (Figure 2; Additional file 3 and Additional file 4). In addition, DETs involved in carbohydrate and energy metabolisms, fatty acid metabolism, cell wall loosening and extension and SCW synthesis were identified and discussed below (Figures 3, 4; Additional file 5 and Additional file 6).

\section{Validation of microarray data}

To validate the microarray data, quantitative real-time polymerase chain reaction (qRT-PCR) analysis was performed on 28 DETs, belonging to various functional categories, during fibre development stages $(0,5,10,15$ and $20 \mathrm{dpa}$ ). The qRT-PCR analysis and microarray data showed similar gene expression pattern for all the genes studied (Figure 5, Additional file 7).

\section{Differentially expressed transcription factors}

DETs encoding transcription factors (TFs) belonging to various families were identified in the $f l$ mutant in all stages of ovule development as compared to WT (Figure 2; Additional file 3). TFs belonging to the AP2-EREBP, C2H2, NAC and WRKY were highly down-regulated at fibre initiation $(0 \mathrm{dpa})$ and early elongation stages ( $5 \mathrm{dpa}$ ) in the $f l$ mutant. Similarly, TFs belonging to the heat shock transcription factor (HSF) family were down-regulated at 10 and $20 \mathrm{dpa}$ in the $f l$ mutant (Figure 2). Further, several transcripts encoding MYB family TFs were differentially expressed at 0 and $10 \mathrm{dpa}$. In addition, transcripts encoding integrase-type DNA-binding superfamily proteins involved in defence mechanism were highly down-regulated at 0 and $10 \mathrm{dpa}$ suggests their involvement in fibre development (Figure 5A1). Transcripts encoding AP2-EREBP family TFs such as ethylene responsive element binding factors (ERFs), redox responsive transcription factor 1 (RRTF1, Figure 5A2) and DREB1-like (DREBP1L) were highly down-regulated at fibre initiation $(0 \mathrm{dpa})$ and elongation ( $5 \mathrm{dpa}$ ) stages in the $f l$ mutant. In addition, transcripts encoding salt tolerance zinc finger (STZ) TFs belonging to $\mathrm{C} 2 \mathrm{H} 2$-type zinc finger family and heat shock transcription factor 2 (HSFA2) were downregulated at fibre initiation and elongation stages, respectively (Figure 5A3).

Transcripts encoding R2R3-MYB transcription factors such as GhMYB25/AtMYB16 (Figure 5A4), AtMYB106 and $A t M Y B 73$ were down-regulated at fibre initiation and elongation stages. It has been shown that GhMYB25 [14] and GhMYB25-like [16] are highly expressed during fibre initiation stage and have a role in regulation of fibre cell elongation and trichome development. AtMYB60 has been shown to be involved in root growth of Arabidopsis under drought stress [31]. Further, 


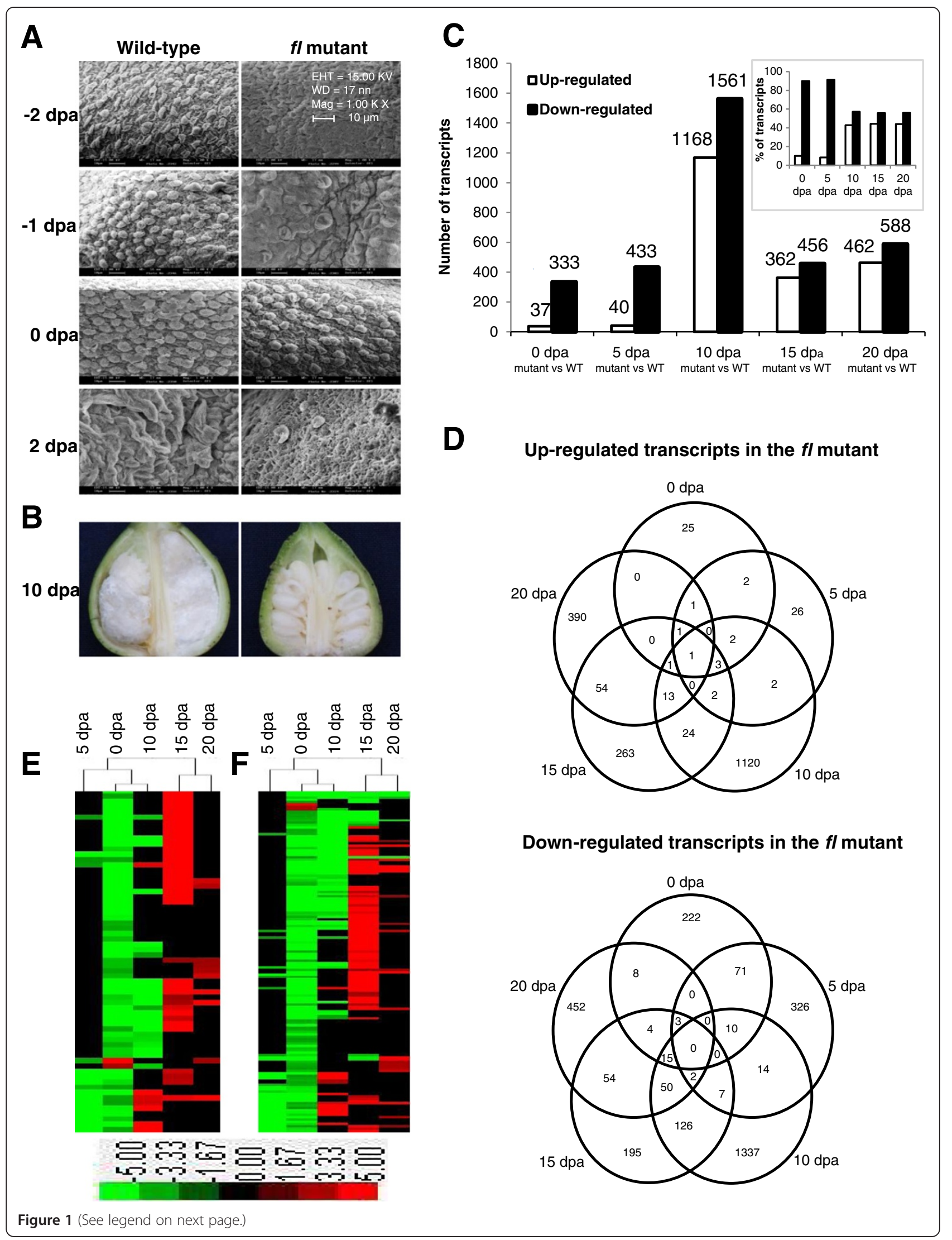


(See figure on previous page.)

Figure 1 Scanning electron microscopy (SEM) and transcriptome analyses during fibre development stages in G. hirsutum L. cv. MCU5 wild-type and its near isogenic fuzzless-lintless (fl) mutant. (A) SEM images (bar is $10 \mu \mathrm{M}$ ) of epidermal layer of -2 to 2 dpa ovules from the WT and fl mutant. (B) Fibre-bearing ovules of WT and fuzzless-lintless ovule of mutant at $10 \mathrm{dpa}$. (C) Number of differentially expressed transcripts (DETs) during fibre development stages in the $\mathrm{fl}$ mutant as compared to their respective stages in WT and inset represents the percentage of up- and down-regulated transcripts in the $f$ mutant (D) Venn diagrams showing the commonly up- and down-regulated transcripts among the fibre development stages in the $\mathrm{fl}$ mutant. Cluster analysis showing the down-regulation of transcripts related to transcription factors (E) and phytohormones (F) at 0 dpa and up-regulation of about $50 \%$ of those transcripts at $15 \mathrm{dpa}$. DETs with $p$ value $\leq 0.01$ and fold change $\geq 3$ were included and presented in Additional file 1.

transcripts encoding NAC family TFs were downregulated at $0 \mathrm{dpa}$ (NAC047) and early elongation (NAC047, NAC072/RD26 and NAC074) stages. Another stress responsive gene, multiprotein bridging factor $1 \mathrm{C}$ (MBF1c) was down-regulated at 10 and $20 \mathrm{dpa}$. It has been reported that MBF1c is involved in biotic and abiotic stress tolerance by activating ethylene-responsive signal transduction pathway [32]. In addition to stress responsive TFs, transcripts encoding the homeodomain proteins such as (HOX3)/glabrous 11 and knotted1-like homeobox gene 3 (KNAT3) involved in Arabidopsis trichome and root development, respectively were down-regulated at $10 \mathrm{dpa}$.

The developing fibre cell during initiation and elongation stages accumulate a large amount of solutes in order to maintain the required turgor pressure. Expression of stress responsive factors during initiation and elongation stages suggests stress-like condition being generated due to turgor pressure. The stage specific transcriptome analysis showed the down-regulation of several stress responsive TFs in the $f l$ mutant during the initiation and elongation stages as compared to WT. This could be due to the fact that these TFs may not have any role in the $f l$ mutant as there is no further development of the fibre initials after $2 \mathrm{dpa}$. In contrast, our data showed the up-regulation of about $50 \%$ of the same TFs at much later stage (15 dpa) in the $f l$ mutant (Figure 1E). The down-regulation of stress responsive TFs in the initiation stage and up-regulation of the same factors at $15 \mathrm{dpa}$ in the $f l$ mutant as opposed to WT suggests the loss of coordination among the regulatory mechanisms and the factors involved in the stress response in the mutant.

\section{Calcium and phytohormones-mediated signalling}

Calcium mediated signalling plays an important role in cell division and differentiation including root hair elongation [33]. Preferential expression of genes encoding calcium binding proteins involved in $\mathrm{Ca}^{2+}$-mediated signalling pathways during fibre initiation and elongation

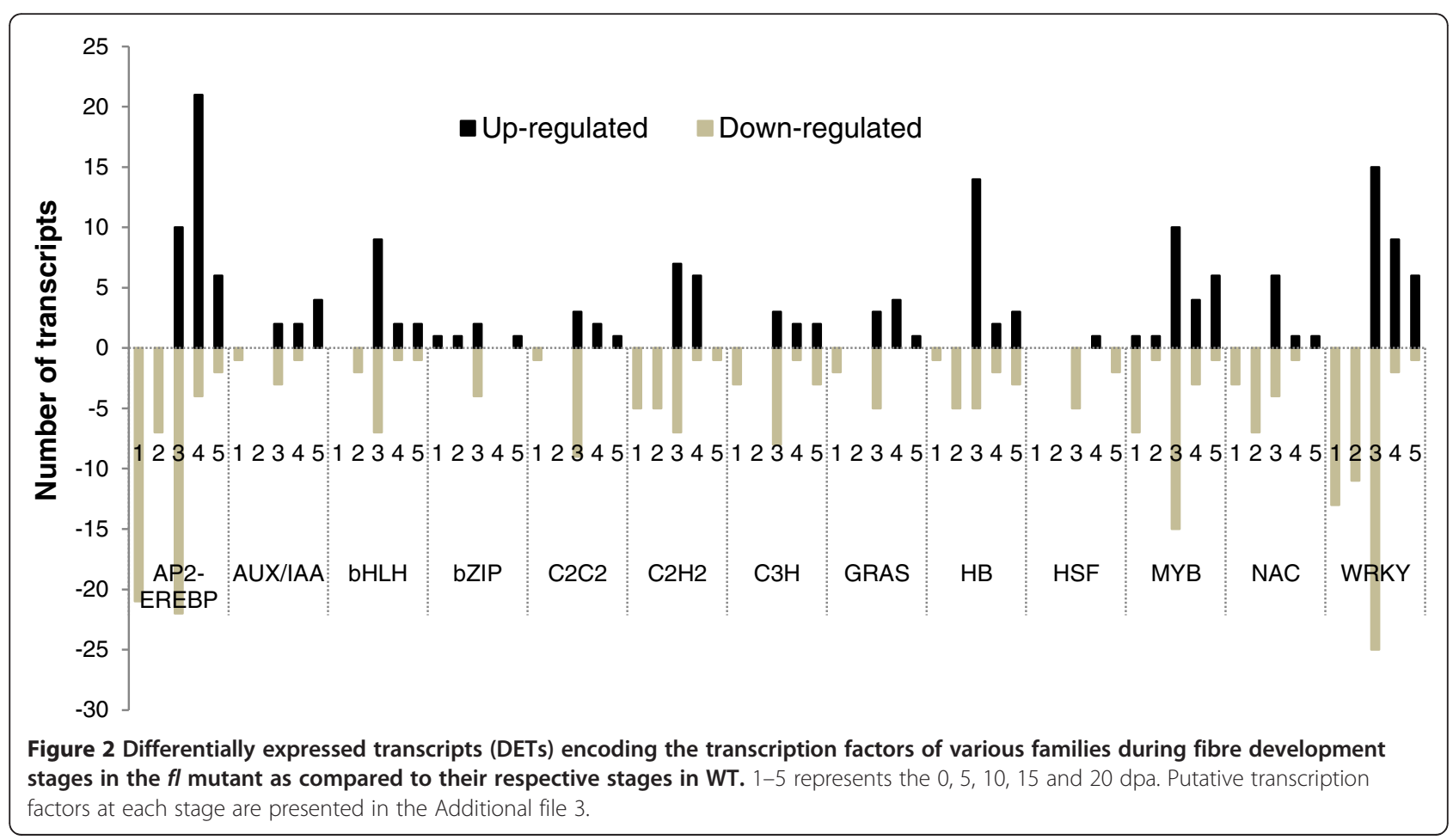


A

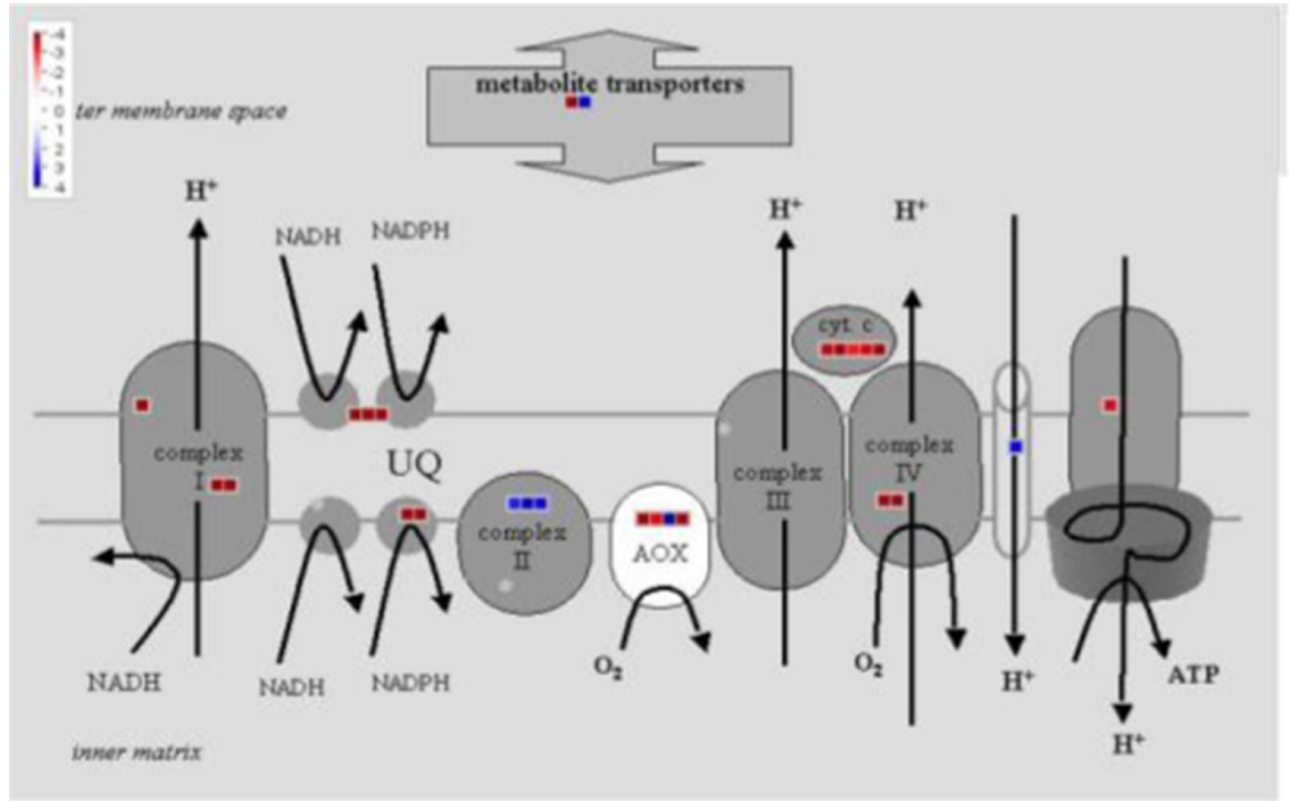

B

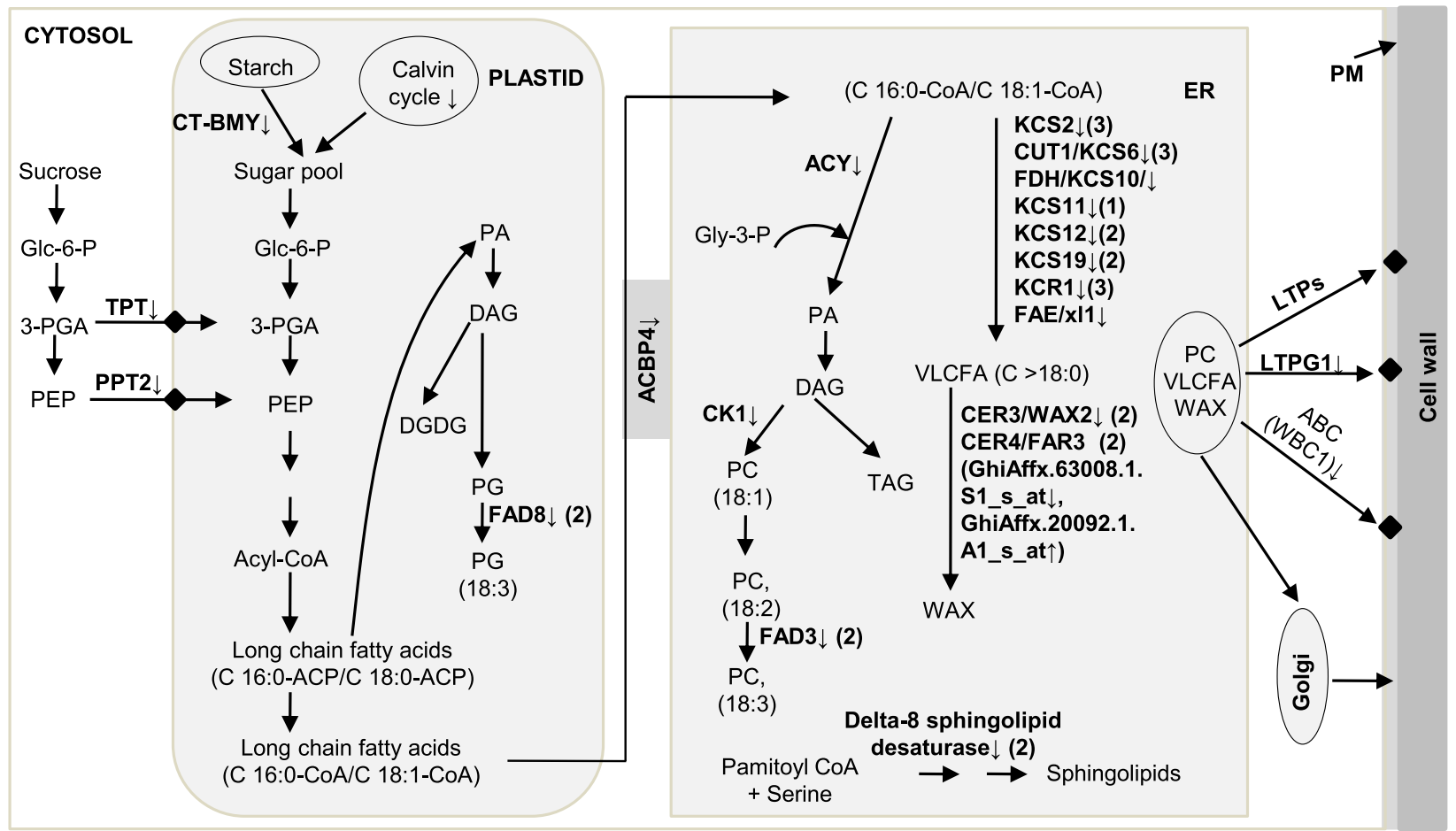

Figure $\mathbf{3}$ (See legend on next page.) 
(See figure on previous page.)

Figure 3 Differentially expressed transcripts (DETs) involved in mitochondrial electron transport system (mETS) and fatty acid metabolism in the $\mathrm{fl}$ mutant as compared to WT at $10 \mathrm{dpa}$. (A) MapMan-based visualization of the DETs involved in mETS in the $\mathrm{fl}$ mutant. Small blue and red colour squares represents up- and down-regulated transcripts, respectively. (B) Over view of DETs involved in fatty acid metabolism in the $\mathrm{fl}$ mutant. ABC: ATPase transporter, ACBP: acyl-CoA binding protein, ACY: acyltransferase-like protein, CER: eceriferum/fatty acid hydroxylase, CK: choline kinase, CT-BMY: chloroplast beta-amylase, DAG: diacylglycerol, DGDG: digalactosyldiacylglycerol, FAD: fatty acid desaturase, FAE: Long chain fatty acid elongation enzyme, FDH: Fiddlehead-like protein, Glc-6-P: glucose 6-phosphate, Gly-3-P: Glycerol-3phosphate, KCS: beta-ketoacyl-CoA synthase, KCR: beta-ketoacyl reductase, LTP: lipid transfer protein, LTPG1: glycosylphosphatidylinositolanchored lipid protein transfer1, PA: phosphatidic acid, PC: phosphatidylcholine, PEP: phosphoenol pyruvate, PG: phosphatidylglycerol, 3-PGA: phosphoglyceric acid, PM: plasma membrane, PPT2: phosphoenolpyruvate (PEP)/phosphate translocator 2, TAG: triacylglycerol, TPT: glucose-6phosphate/phosphate translocator-related and VLCFA: very long chain fatty acids. DETs related to mETS and fatty acid metabolism are presented in the Additional file 6.

stages have been reported in cotton [33,34]. In this study, several transcripts coding for calcium-binding EFhand family proteins were highly down-regulated at 0 and $10 \mathrm{dpa}$ in the $f l$ mutant as compared to WT (Additional file 1; Figure 5B1, 5B2, 5B3). In addition, transcripts encoding $\mathrm{Ca}^{2+}$-dependent protein kinase 6 (CDPK6) and glutamate decarboxylase (GAD) were down-regulated at 0 and $10 \mathrm{dpa}$, respectively. Further, transcripts encoding $\mathrm{Ca}^{2+}$-ATPase that was shown to play a role in maintaining the calcium homeostasis in the cell [35] (Figure 5B4) and C2 calcium-dependent membrane targeting protein were highly down-regulated at 0 and $10 \mathrm{dpa}$ in the $f l$ mutant. These results are in agreement with the earlier reports that suggested a role for $\mathrm{Ca}^{2+}$-mediated signal transduction and $\mathrm{Ca}^{2+}$-homeostasis in the fibre cell initiation and elongation [33,34].

Phytohormones play an important regulatory role in various plant growth and developmental processes through intracellular signalling events leading to welldefined changes in the gene expression. In the present study, DETs involved in phytohormone biosynthesis and signal transduction pathways were identified at different stages in the $f l$ mutant as compared to their respective stages in WT (Additional file 4). Ethylene [9], auxin [11] and brassinosteroids (BR) [12] were shown to play a role in fibre cell initiation and elongation. In this study genes involved in the phytohormone signal transduction pathways and the biosynthesis of auxin, BR, ethylene,

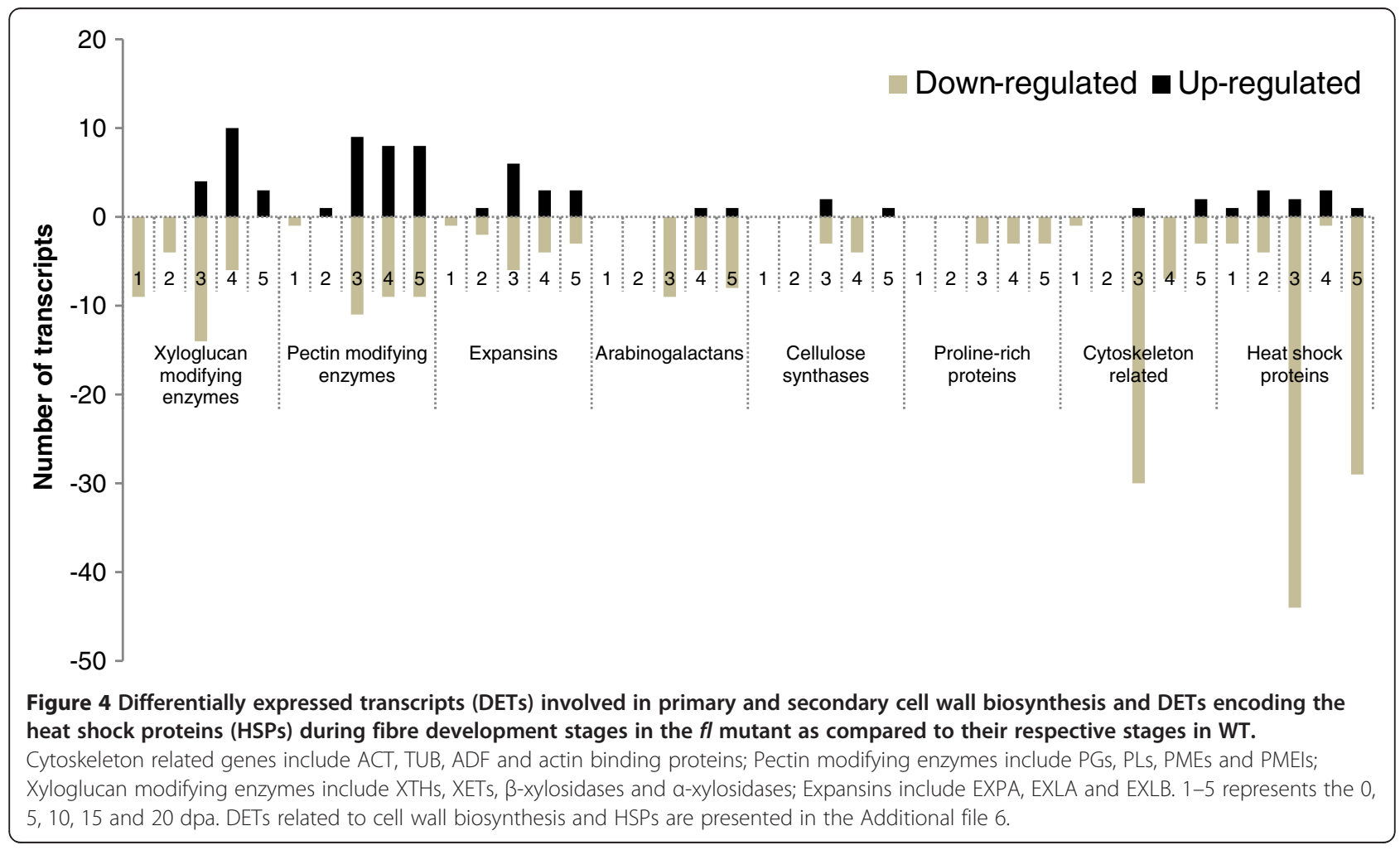




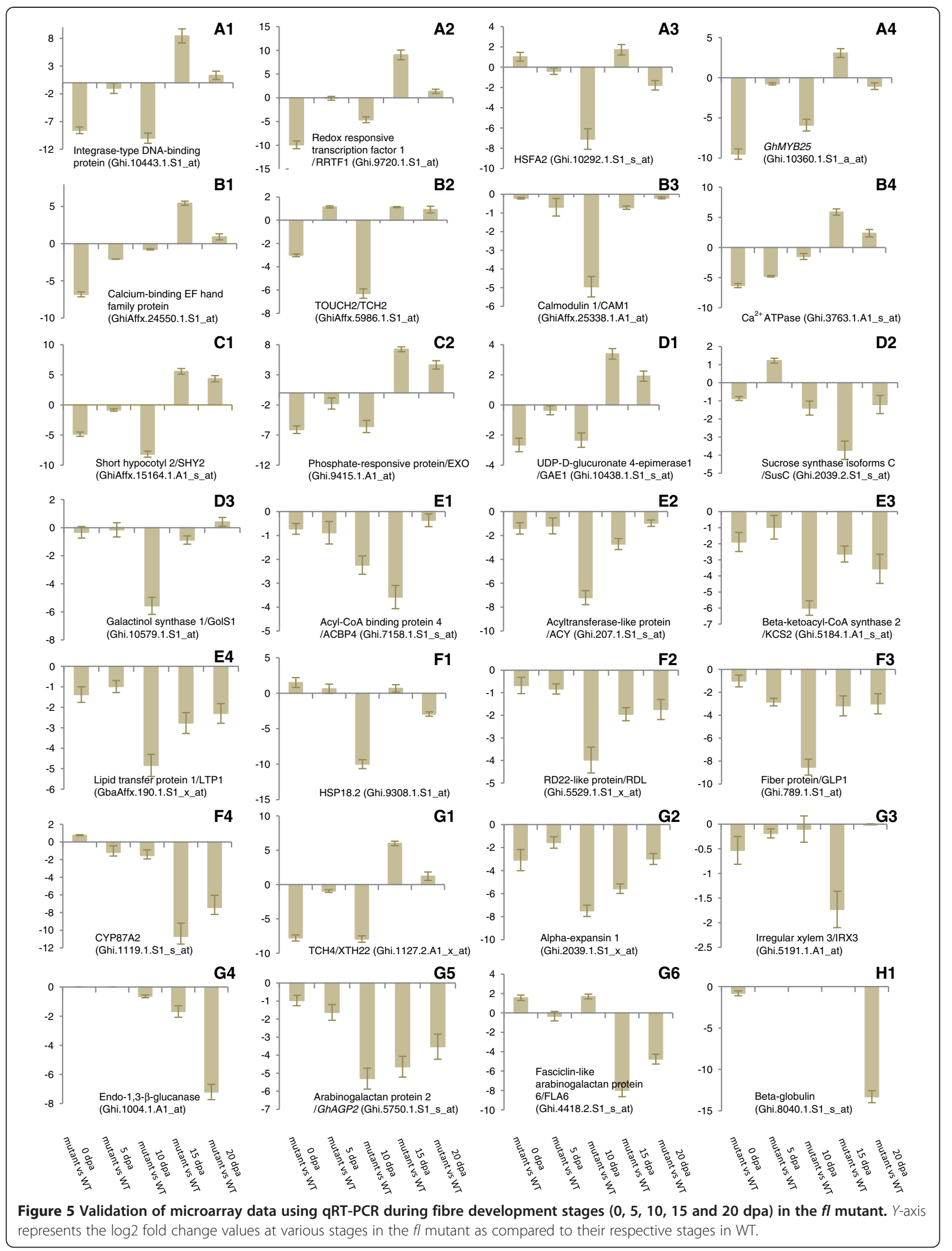


gibberellic acid (GA), were found to be down-regulated at fibre initiation and elongation stages in the $f l$ mutant (Additional file 4).

While the transcripts encoding cytochrome P450 83B1 (CYP83B1) were down-regulated at 0 and $5 \mathrm{dpa}$, the transcript encoding CYP79B2 was highly downregulated at $10 \mathrm{dpa}$. CYP79B2 catalyzes the conversion of tryptophan to Indole-3-acetaldoxime and CYP83B1/ RNT1/SUR2 catalyzes the first step in the biosynthesis of indole glucosinolates from indole-3-acetaldoxime and regulates the level of IAA. In addition, a transcript encoding the P-glycoprotein 19 (PGP19) involved in auxin transport was down-regulated at $10 \mathrm{dpa}$ in the $f l$ mutant [36]. Furthermore, transcripts encoding the auxin responsive gene hypocotyls 2 (SHY2/AUX2-11/IAA4) (Figure $5 \mathrm{C} 1$ ) were highly down-regulated at 0 and 10 dpa. Also, auxin-induced in root cultures 1 (AIR1) were highly down-regulated at 15 and $20 \mathrm{dpa}$. Transcripts encoding ACC oxidases (ACOs), involved in ethylene biosynthesis were down-regulated at $0 \mathrm{dpa}(\mathrm{ACO} 2$ and ACO4) and 5 dpa (ACO1, ACO2, ACO3 and ACO4/ $\mathrm{EFE})$ in the $f l$ mutant. Along with ACC oxidases, a transcript encoding ACC synthase 6 (ACS6) which is a rate limiting enzyme in ethylene biosynthesis was also downregulated at $0 \mathrm{dpa}$. These results suggest the downregulation of auxin and ethylene biosynthesis at fibre initiation and early elongation stages in the $f l$ mutant.

Transcripts encoding the enzymes involved in BR biosynthesis such as 3-oxo-5- $\alpha$-steroid 4-dehydrogenase family protein (DET2) [13] and sterol methyltransferase 2 (SMT2) were down-regulated at $10 \mathrm{dpa}$ in the $f l \mathrm{mu}-$ tant. In addition, a number of genes (eg. BRI1, BRH1 and P450-like protein/CYP734A1/BAS1) involved in BR signalling pathway [37] was also differentially expressed at various stages in the $f l$ mutant as compared to WT (Additional file 4). Down-regulation of several genes involved in ethylene, auxin and BR biosynthesis and signal transduction pathways during fibre initiation and elongation stages suggested their regulatory role in fibre cell initiation and elongation. In the present study we have identified a number of genes involved in the phytohormone biosynthesis and signal transduction pathways that were down regulated at fibre initiation stage (0 dpa) in $f l$ mutant as compared to WT. Interestingly, $~ 50 \%$ of these genes were found to be up-regulated at late elongation stage $(15 \mathrm{dpa})$ in the $f l$ mutant (Figure $1 \mathrm{~F})$, suggesting the delay in the phytohormone stimulation and signalling in the mutant.

\section{Carbohydrate and energy metabolism}

Sugars are the basic source of energy and carbon skeletons for all biomolecules and they are required for the regulation of cell homeostasis and synthesis of cell wall precursors. UDP-D-glucose (UDP-Glc) is a central metabolite in carbohydrate metabolism and is the common precursor for synthesis of cell wall polysaccharides such as pectin, hemicellulose and cellulose. In the present study, transcripts encoding the enzymes, involved in synthesis of cell wall precursors, such as UDP-glucose pyrophosphorylases (UGP1 and UGP2), UDP-glucose 6dehydrogenase (UGD), UDP-D-glucuronate 4-epimerase 1 (GAE1), UDP-XYL synthase 5 (UXS5) were downregulated during fibre elongation stage in the $f l$ mutant. UGP and UGD catalyse the formation of UDP-Dglucose and UDP-D-glucuronic acid, respectively whereas GAE1 (Figure 5D1) and UXS5 catalyse the formation of UDP-D-galacturonic acid (precursor for pectin) and UDP-D-xylose (precursor for hemicellulose) from UDP-D-glucuronic acid, respectively (Additional file 5 and Additional file 6). The UDPglucose formed from the sucrose by sucrose synthase (Sus) is used directly as a substrate by the cellulose synthase complex. The Sus isoform C (SusC) involved in cellulose synthesis was highly down-regulated at 15 $\mathrm{dpa}$ in the $f l$ mutant (Figure 5D2, Additional file 5 and Additional file 6) [38]. Carbohydrates such as raffinose family oligosaccharides (RFOs) are the main storage forms of carbohydrates in the seeds, which confer desiccation tolerance. In the present study, transcripts encoding galactinol synthase (GolS) that catalyse the first committed step in the biosynthesis of RFOs (GolS1 and GolS2) were highly downregulated at $10 \mathrm{dpa}$ in the $f l$ mutant (Figure 5D3; Additional file 5 and Additional file 6). Further, raffinose synthase (RS/SIP1), stachyose synthase (STS) were also down-regulated. Trehalose is another storage carbohydrate that was shown to be involved in desiccation tolerance [39]. Transcripts encoding the enzymes involved in trehalose biosynthesis such as trehalose-6-phosphate synthase (TPS) and trehalose-6phosphate phosphatase (TPP) were down-regulated at fibre elongation stage. Previously we have shown that, both GolS and TPS were highly up-regulated under drought stress at fibre elongation stage suggesting the role of these genes in fibre development [40].

In the present study, transcripts encoding the enzymes involved in Calvin cycle such as ribulose bisphosphate carboxylase, glyceraldehyde 3-phosphate dehydrogenase, transketolase and fructose-bisphosphate aldolase (FBPA) were down-regulated at 10 and $15 \mathrm{dpa}$ thus indicating the reduction of sugar pool in the $f l$ mutant ovules (Additional file 6). Mitochondrial electron transport system (mETS) is the main pathway for the generation of ATP by oxidative phosphorylation. Recently it has been shown that the ATP synthase $\delta 1$ subunit (GhATP $\delta 1)$ is required for mitochondrial ATP synthesis and to maintain higher ATP/ADP ratio which facilitates faster fibre cell elongation [21]. Several transcripts encoding the 
enzymes involved in mETS were down-regulated in the $f l$ mutant which in turn decreases the $\mathrm{NAD}^{+} / \mathrm{NAD}(\mathrm{P}) \mathrm{H}$ ratio and efficiency of mitochondrial ATP production (Figure 3A; Additional file 6). The ATP/ADP and $\mathrm{NAD}^{+} / \mathrm{NAD}(\mathrm{P}) \mathrm{H}$ ratios are very critical for maintaining redox-homeostasis and functioning of several enzymes involved in synthesis of cell wall precursors and fatty acids [41]. These data suggest the importance of interconversion of sugars and the role of mETS during fibre elongation process.

\section{Fatty acid metabolism}

Fatty acid biosynthesis is another important biochemical pathway involved in fibre cell development $[5,42,43]$. In the present study, transcripts encoding the enzymes involved in the biosynthesis of very long chain fatty acids (VLCFAs), cuticular wax and phospholipids were down-regulated at fibre elongation stage in the $f l \mathrm{mu}-$ tant as compared to WT. Calvin cycle and sugars exported from the cytosol are main source of sugar pool for synthesis of fatty acids in plastids of nonphotosynthetic tissue. Fatty acids synthesized within the plastids are exported as acyl-CoA esters (C16:0 CoA and $\mathrm{C} 18: 1 \mathrm{CoA}$ ) to the endoplasmic reticulum (ER) for synthesis of VLCFA that are precursors for synthesis of phospholipids, sphingolipids, wax and cuticular matrix (Figure 3B; Additional file 6). Transcripts encoding the sugar transporters such as triose phosphate/phosphate translocator (TPT) and phosphoenolpyruvate (PEP)/ phosphate translocator (PPT) and acyl-CoA binding protein 4 (ACBP4, Figure 5E1) were down-regulated at $10 \mathrm{dpa}$ in the $f l$ mutant. Glucose-6-phosphate/phosphate translocator (GTP), TPT and PPT are required for import of cytosolic Glc-6-P, 3-PGA and PEP respectively, from cytosol into the plastid for synthesis of acylCoA [44]. Cytosolic ACBP4 and ACBP5 were shown to bind long-chain acyl-CoAs and involved in the trafficking of oleoyl-CoA from the plastids to ER for synthesis of VLCFAs [45].

Phospholipids are major structural components of plasma membrane (PM) and involved in lipid signalling pathway. PM plays a crucial role during fibre cell elongation. Phosphatidic acid (PA) is the key component in phospholipid biosynthesis. The transcripts encoding acyltransferase-like protein (ACY) involved in synthesis of PA in ER (Figure 5E2) and AGC (cAMP-dependent, cGMP-dependent and protein kinase C) kinase family proteins (AGC2-1 and AGC2-2) were highly downregulated at $10 \mathrm{dpa}$ in the $f l$ mutant. Further, transcripts encoding fatty acid desaturases (FADs) such as FAD8 (plasticidal) and FAD3 (ER) and delta-8 sphingolipid desaturase were down-regulated. In lipid-signalling pathway PA specifically binds to AtPDK1 (3-phosphoinositide-dependent protein kinase 1) and stimulates AGC2-1 and it has been shown that agc2-1 knockout mutants resulted in reduced root hair length suggesting its role in cell elongation [46]. Down-regulation of genes involved in phospholipid biosynthesis and lipid signalling suggested their role in fibre development.

Transcripts encoding beta-keto acyl-CoA synthases (KCS2, KCS6/CER6/CUT1, KCS10/FDH, KCS11, KCS12 and KCS19) and beta-keto acyl reductase (KCR1/ YBR159), catalyzing the first two committed steps in VLCFA synthesis, were highly down-regulated at fibre elongation stage in the $f l$ mutant (Figures 3B, 5E3). In addition, a transcript encoding a long chain fatty acid elongation enzyme (ELo2/SUR4) was highly downregulated at 5, 10 and $20 \mathrm{dpa}$. A transcript encoding epoxide hydrolase involved in cutin layer biosynthesis was highly down-regulated at $10 \mathrm{dpa}$ [47].

Lipid transfer proteins (LTPs) are involved in transport of lipids from ER to PM and the subsequent transport of lipids from PM to the cell exterior appears to be carried out by PM localized transporters such as ATP-binding cassette $(\mathrm{ABC})$ transporters $[48,49]$. In the present study, several transcripts encoding LTPs (Figure 5E4), ABC transporters such as white/brown complex proteins (WBC1 and WBC12/CER5) were down-regulated at fibre elongation stage in the $f l$ mutant. Further, a transcript encoding glycosylphosphatidylinositol-anchored lipid protein transfer 1 (LTPG1) was down-regulated. High level expression of WBC1 during fibre elongation and plasma membrane localization was reported in upland cotton [50]. In $A$. thaliana WBC12/CER5 and WBC11 have been shown to be involved in cuticular lipid transport [51] and the localization of LTPG in PM and its role in lipid deposition has been demonstrated [52]. Down-regulation of genes involved in fatty acid metabolism in the $f l$ mutant particularly at elongation stage suggests the importance of these genes in fibre cell development.

\section{Transcripts involved in regulation of osmotic potential, ion-homeostasis and protein stabilization}

Cotton fibre elongates through diffuse growth mechanism and turgor-driven pressure is a significant driving force for cell enlargement $[17,18,53,54]$. The turgordriven pressure largely depends on accumulation of osmolytes and ion-homeostasis $[17,18]$. Further, genes involved in ROS homeostasis and protein stabilization play a crucial role during fibre development $[6,8]$. In the present study several transcripts involved in these processes were differentially expressed at various fibre development stages in the $f l$ mutant as compared to WT. Transcripts encoding the ion and sugar transporters (NRT1.5, VIT and STP1) and membrane intrinsic proteins (PIP2;2 and $\gamma$-TIP1;3) were highly down-regulated during fibre elongation stage in the $f l$ mutant (Table 1 , 
Table 1 Some of the differentially expressed stress responsive transcripts at various fibre development stages in the $\mathrm{fl}$ mutant as compared to their respective stages in WT

\begin{tabular}{|c|c|c|c|c|c|c|}
\hline Probe set ID & $0 \mathrm{dpa}$ & 5 dpa & 10 dpa & $15 \mathrm{dpa}$ & $20 \mathrm{dpa}$ & Annotation \\
\hline Ghi.10292.1.S1_s_at & & & -108.2 & & -6.7 & Heat shock transcription factor A2 (HSFA2) \\
\hline Ghi.9308.1.S1_at & 4.2 & & -142.5 & & -6.5 & Heat shock protein HSP18.2 \\
\hline GhiAffx.3815.1.A1_s_at & & & -62.9 & & -10.9 & HSP90 \\
\hline Ghi.8378.2.S1_s_at & & & -36.1 & & -5.8 & HSP70 \\
\hline GhiAffx.10920.2.S1_at & & -11.9 & & & & Plasma membrane intrinsic protein 2;2 (PIP2;2) \\
\hline Ghi.4613.1.S1_at & & & -36.5 & -6.7 & & Gamma-tonoplast intrinsic protein 1;3 $(\gamma$-TIP1;3) \\
\hline Ghi.3135.1.S1_at & & -62.5 & & & & Nitrate transporter 1.5 (NRT1.5) \\
\hline Ghi.6855.1.A1_s_at & & -6.5 & & & & Proline transporter 2 (PROT2) \\
\hline Ghi.9885.1.A1_at & & -4.6 & & & & Sugar transporter 1 (STP1) \\
\hline GhiAffx.17156.1.S1_at & & & -22.7 & & & Vacuolar iron transporter (VIT) \\
\hline Ghi.10646.1.S1_s_at & & -5.2 & 178.7 & & 102.2 & Osmotin 34 \\
\hline GarAffx.24961.2.S1_s_at & -3.3 & & -22.3 & -8 & & Responsive to desiccation 22 (RD22) \\
\hline Gra.2810.2.A1_s_at & -3.2 & & -21.8 & -8.2 & & $\mathrm{RD} 22$ \\
\hline Ghi.5529.1.S1_x_at & -3 & & -35 & -8.2 & -4.4 & RD22-like protein (RDL) \\
\hline Ghi.779.1.S1_at & & -20.5 & & & & Glutathione S-transferase 30 (GST30) \\
\hline Ghi.7950.1.S1__at & & -14 & & & & Peroxidase 2 (PA2) \\
\hline Ghi.10450.1.S1__s_at & -4.8 & & -13.3 & 7 & & Thioredoxin family protein (GRX480) \\
\hline Gra.2231.1.S1_s_at & -13.2 & -26.2 & 27.4 & & & Asparagine synthase 1 (ASN1) \\
\hline Ghi.789.1.S1_at & & & -147.1 & -21.3 & -10.5 & Germin-like protein (GLP1)/Fibre protein \\
\hline Ghi.6103.1.s̄̄_at & & & -28.8 & -9.4 & -3.2 & Chalcone synthase (CHS) \\
\hline Gra.432.1.S1_s_at & -19.8 & -5.1 & & -4.6 & 17.7 & $\mathrm{CHS}$ \\
\hline Ghi.3763.1.A1_s_at & -52.9 & -16.5 & & 28.4 & 3.7 & $\mathrm{Ca}^{2+}$-ATPase \\
\hline GhiAffx.3680.3.A1_at & & & -34.4 & & & Suppressor of $\mathrm{K}^{+}$transport growth defect-like protein (SKD1) \\
\hline
\end{tabular}

Figure 5F1-5F4). A transcript encoding SKD1 (suppressor of $\mathrm{K}^{+}$transport growth defect 1) which is an AAAtype ATPase family protein was highly down-regulated at $10 \mathrm{dpa}$ in the $\mathrm{fl}$ mutant. The expression of $m c S K D 1$ (homologous to AtSKD1) in elongating root tips and its role in endoplasmic reticulum-Golgi mediated protein sorting machinery and $\mathrm{K}^{+}$uptake has been demonstrated [55]. Further, transcripts encoding heat shock proteins (sHSPs, HSP70 and HSP90) were highly down-regulated at 10 and $20 \mathrm{dpa}$ in the $f l$ mutant.

\section{Primary and secondary cell wall biosynthesis}

Several transcripts involved in the primary and secondary cell wall biosynthesis were differentially expressed at various stages of fibre development in the $f l$ mutant as compared to their respective stages in WT (Figure 4; Additional file 6). A large number of genes involved in primary cell wall biosynthesis and elongation such as those coding for xyloglucanases, pectinases, expansins and arabinogalactans were down-regulated at $15 \mathrm{dpa}$. The role of xyloglucan modifying enzymes such as xyloglucan endotransglycosylases (XETs) and xyloglucan endotransglucosylase/hydrolases (XTHs) has been well established in fibre cell development $[3,22]$. In the present study, transcripts encoding TOUCH 4 (TCH4)/ XTH22 (Figure 5G1) and XTH23/XTR6 were highly down-regulated at initiation and elongation stages in the $f l$ mutant. Further, transcripts encoding endo-xyloglucan transferase/AtXTH7 involved in fibre elongation were highly down-regulated at 10 and $15 \mathrm{dpa}$. These data suggested their role in primary cell wall synthesis and fibre development. Pectins are major components of the primary cell wall and in cotton pectins constitute about $25 \%$ of the cell wall of cotton fibre. Thus, pectin modifying enzymes play a major role in the fibre cell wall development [56]. Several transcripts encoding pectin modifying enzymes such as poly galacturonases (PGs), pectate lyases (PLs), pectin methyl esterases (PMEs) and pectin methyl esterase inhibitors (PMEIs) were differentially expressed during fibre elongation stage in the $f l$ mutant as compared to WT (Figure 4; Additional file 6). Further, large number of transcripts encoding glycosyl hydrolases and transferases were down-regulated at 10 and $15 \mathrm{dpa}$. Particularly, transcripts encoding $\beta$ galactosidase 13 (BGAL13) and UDP-glucosyl transferase 74B1 (UGT74B1) were highly down-regulated at 10 $\mathrm{dpa}$ in the $f l$ mutant. Among the glycosyl hydrolases, galactosidases (GALs) catalyzes the removal of nonreducing $\beta$-D-galactosyl residues from $\beta$-D-galactosides. It is therefore thought that GALs may be one of the main enzymes responsible for the metabolism of galactose rich polymers such as galactan and arabinogalactan and galactose containing side chains of cell wall polysaccharides $[3,5]$.

Expansins are highly expressed in cotton fibre tissue and play an important role in cell wall loosening during fibre elongation stage [3,24]. A transcript (Ghi.2039.1. S1_x_at/AY189969.1) encoding $\alpha$-expansin 1 (EXPA1) was highly down-regulated at 10,15 and $20 \mathrm{dpa}$ in the $f l$ mutant (Figure 5G2; Additional file 6). Further, transcripts encoding expansin-like B1 (EXLB1) were highly down-regulated at 0 and $5 \mathrm{dpa}$ and expansin-like $\alpha$ (EXLA) were down-regulated at $10 \mathrm{dpa}$. These data suggest that both expansins and expansin-like genes play 
crucial role in fibre cell development. The transcripts encoding cellulose synthases were down-regulated at 15 dpa at which PCW synthesis ceases and cellulose synthesis begins (Figure 5G3; Additional file 6). Further, a transcript encoding endo-1,3- $\beta$-glucanase was highly downregulated at $20 \mathrm{dpa}$ in the $f l$ mutant (Figure 5F4). It was reported that the expression of endo-1,3- $\beta$-glucanase, which is very low at the fibre elongation stage (5-12 dpa) and increases gradually during secondary cell wall formation in G. hirsutum (15-20 dpa), is involved in the degradation of callose and opening of the plasmodesmata during cellulose deposition $[5,19]$. Interestingly, a transcript encoding trichome birefringence (TBR) like 38 (TBL38) gene was highly down-regulated at $5 \mathrm{dpa}$ and 15 dpa. Recently, it has been shown that TBL genes are involved in secondary cell wall synthesis by influencing the esterification state of pectic polymers and increasing the crystalline cellulose in Arabidopsis [57]. Along with the cell wall modifying enzymes, several transcripts encoding structural proteins such as AGPs (GhAGP2), FLAs (GhFLA6/AtFLA11 and GhFLA3/AtFLA7) and proline-rich proteins (PRPs; PRP3 and PRP5) were down-regulated at fibre elongation and $\mathrm{SCW}$ synthesis stages in the $f l$ mutant (Figures 4, 5G5, 5G6; Additional file 6).

Actin cytoskeleton plays an important role in fibre elongation and onset of secondary cell wall deposition $[6,26,27]$. Tubulins (TUBs) are major components of cytoskeleton microtubules and play a vital role in cell expansion by controlling the orientation of cellulose microfibrils [25]. In the present study, transcripts encoding actins (ACTs), profilin, ATP binding microtubule motor family proteins, actin depolymerizing factor (ADF) and $\mathrm{Xu}-142$ beta-tubulin1 were down-regulated at $10 \mathrm{dpa}$ in the $f l \mathrm{mu}-$ tant (Figure 4, Additional file 6). The down-regulation of several genes involved in cell wall loosening and elongation, structural reinforcement and cytoskeleton dynamics suggests their role in fibre development.

\section{Conclusions}

SEM study indicated a delay in the initiation process of fibre cells on the epidermal layer of ovules in the $f l \mathrm{mu}-$ tant and elongation of these initials was completely stopped at about $2 \mathrm{dpa}$. Transcriptome analysis revealed the differentially expressed transcripts at various fibre developmental stages $(0,5,10,15$ and $20 \mathrm{dpa})$ in the $f l$ mutant as compared to their respective stages in the wild-type. The down-regulation of stress responsive TFs (representing 19.6\% of total down-regulated transcripts) and transcripts involved in the $\mathrm{Ca}^{2+}$-mediated signal transduction and phytohormone (ethylene, auxin and $\mathrm{BR})$ biosynthesis and signalling pathways at fibre initiation stage suggested their regulatory role in fibre cell initiation and differentiation. Further, down-regulation of transcripts involved in the Calvin cycle, synthesis of RFOs (GolS1, GolS2), trehalose (TPS and TPP) and cell wall precursors and mitochondrial electron transport system at fibre elongation stage in the $f l$ mutant suggested the reduction of overall sugar pool and significance of mitochondrial energy supply required for the synthesis of cell wall polysaccharides and to maintain redox-homeostasis. Similarly, down-regulation of transcripts involved in the synthesis of VLCFAs and phospholipids and lipid transport suggests the reduced fatty acid metabolism in the $f l$ mutant. This study also supports the earlier findings on the role of pectin and xyloglucan modifying enzymes, expansins and arabinogalactans and cytoskeleton dynamics in the cell wall loosening and elongation process. Also the delayed expression of genes involved in phytohormone signalling and stress responsive TFs identified in the present study suggests the lack of coordinated expression of regulatory mechanisms involved in fibre cell initiation and differentiation in $f l$ mutant. On the whole, the comprehensive transcriptome analysis of Gossypium hirsutum L. cv. MCU5 and its near isogenic fuzzless-lintless mutant revealed the stage specific involvement of several genes and pathways during fibre development.

\section{Methods}

\section{Plant material}

The Gossypium hirsutum L. cv. MCU5 (wild-type) and its near isogenic fuzzless-lintless mutant were grown in the field following normal agronomic practices. Flowers were tagged on the day of anthesis and considered as 0 dpa (days post anthesis). Cotton bolls were collected at fibre initiation $(-2,-1,0,1$ and $2 \mathrm{dpa})$, elongation $(5,10$ and $15 \mathrm{dpa}$ ) and secondary cell wall synthesis (20 dpa) stages. The -2 and -1 dpa represent the days before anthesis. Harvested cotton bolls were immediately frozen in liquid nitrogen and stored at $-70^{\circ} \mathrm{C}$ until used for total RNA extraction.

\section{Scanning electron microscopy analysis}

Scanning electron microscopy (SEM) was performed at fibre initiation stage ( -2 to $2 \mathrm{dpa}$ ) to observe the development of fibre initials on epidermal layer of ovules as described by Mir and Channa [58]. SEM was carried out with LEO $435 \mathrm{VP}$ scanning electron microscope (LEO Electron Microscopy Ltd., Cambridge, England) at All India Institute of Medical Sciences (AIIMS), New Delhi, India.

\section{RNA isolation}

Total RNA was isolated from fibre-bearing ovules of wild-type (WT) and fuzzless-lintless $(f l)$ mutant collected at various stages of fibre development $(0,5,10,15$ and 20 dpa). RNA was isolated using Spectrum ${ }^{\mathrm{TM}}$ Plant Total RNA kit (Sigma, USA) according to the manufacturer's 
protocol. During RNA purification on-column DNase treatment was given for removing trace amount of DNA. The quality and quantity of total RNA was assessed by Agilent Bioanalyzer 2100 (Agilent Technologies, USA).

\section{Microarray experiments and data analysis}

Affymetrix cotton GeneChip genome array (Affymetrix, USA) having 23,977 probe sets representing 21,854 cotton transcripts was used for transcriptome analysis. Three biological replicates were used to test the reproducibility and quality of the chip hybridization. The One-Cycle Target Labeling and Control Reagent kit was used to prepare biotinylated complementary RNA (cRNA) for microarray hybridization. Array hybridization, staining and washing procedures were carried out as described in the Affymetrix protocols. The arrays were scanned with a GeneChip scanner 3000.

DAT, CEL, CHP, XML and JPEG image files were generated for each array using GeneChip Operating Software (GCOS) platform. CEL files having estimated probe intensity values were analyzed with GeneSpring GX-11.5 software (Agilent Technologies, USA) to get differentially expressed transcripts. The Robust Multiarray Average (RMA) algorithm was used for the background correction, quantile normalization and median polished probe set summarization to generate single expression value for each probe set. Normalized expression values were $\log 2$-transformed and differential expression analysis was performed using unpaired $t$-test. The $p$ values were corrected by applying the false discovery rate (FDR) correction [59]. Differentially expressed transcripts with FDR corrected $p$ value $\leq 0.01$ and fold change $\geq 3$ were included for further data analysis. The DETs were annotated based on NetAffx annotation data for cotton GeneChip (http://www.affymetrix.com). To obtain functional annotation of transcripts, the consensus sequences of probe sets present in the cotton GeneChip genome array were mapped to the Arabidopsis TAIR protein database version 10 (http://www.arabidopsis.org) by BLASTX with $E$ value cut off $\leq \mathrm{e}^{-10}$. Further, the DETs were grouped into various functional categories by using their corresponding Arabidopsis protein IDs in MIPS functional catalogue (http://mips.gsf.de/projects/funcat). To identify the putative transcription factors and transcripts related to phytohormone biosynthesis and signal transduction pathways, the consensus sequences of all probe sets presented in cotton GeneChip genome array were searched against the Arabidopsis transcription factor database (http://plntfdb.bio.uni-potsdam.de, version 3.0) and Arabidopsis hormone database (http://ahd.cbi.pku.edu.cn, version 2.0) respectively, by BLASTX with $E$ value cutoff $\leq \mathrm{e}^{-10}$. Further, MapMan software version 3.5.0 was used to visualize the expression of differentially regulated cotton transcripts onto metabolic pathways (http://gabi.
rzpd.de/projects/MapMan/). The microarray data is deposited in the Gene Expression Omnibus database (GEO, http://www.ncbi.nlm.nih.gov/geo) at the NCBI under the Series Accession numbers GSE38490. The hierarchical clustering was performed based on centroid linkage with $\mathrm{Eu}-$ clidean distance using log fold change data with Cluster 3.0 to display the expression pattern and tree diagram of differentially expressed transcripts [60].

\section{The quantitative real-time PCR (qRT-PCR) analysis}

First strand CDNA was synthesized using $1 \mu \mathrm{g}$ of total RNA using AffinityScript QPCR cDNA Synthesis Kit (Stratagene, Agilent Technologies, USA) according to the manufacturer's instructions. Gene specific qRT-PCR primers were designed using PrimerQuest software (http://eu. idtdna.com) and the list of primers are presented in Additional file 7. The qRT-PCR was performed in triplicates using the Brilliant-III Ultra Fast SYBR Green QPCR master mix in Stratagene MX 3005P (Agilent Technologies, USA) detection system. The GhPP2A1 gene (Accession No: DT545658) from G. hirsutum was used as reference gene to normalize the expression values [61]. The $\log 2$ fold change value was calculated based on $2^{-(\Delta \Delta C t)}$ method.

\section{Additional files}

Additional file 1: List of differentially expressed transcripts (DETs) at $0,5,10,15$ and $20 \mathrm{dpa}$ in the $\mathrm{fl}$ mutant as compared to their respective stages in WT. Excel file containing the DETs with $p$ value $\leq$ 0.01 and fold change $\geq 3$.

Additional file 2: Functional classification of DETs at 0, 5, 10, 15 and 20 dpa in the $f l$ mutant as compared to their respective stages in WT. PPT file containing the DETs grouped into various functional categories based on MIPS data base.

Additional file 3: List of DETs encoding putative transcription factors (TFs) belonging to various families at 0, 5, 10 and 20 dpa in the $f \mathbf{l}$ mutant as compared to their respective stages in WT. Excel file containing the differentially expressed putative transcription factors based on Arabidopsis transcription factor database ( $E$-value cutoff $\leq \mathrm{e}^{-10}$ ).

Additional file 4: List of DETs involved in phytohormone biosynthesis and signalling pathways at 0, 5, 10, 15 and 20 dpa in the $\mathrm{fl}$ mutant as compared to their respective stages in WT. Excel file containing the DETs involved in phytohormone biosynthesis and signalling pathways were identified based on Arabidopsis hormone database ( $E$ value cutoff $\leq \mathrm{e}^{-10}$ ).

Additional file 5: Differentially expressed transcripts (DETs) involved in carbohydrate metabolism at 0, 5, 10, 15 and 20 dpa in the $f l$ mutant as compared to their respective stages in WT. PPT file containing the DETs involved in biosynthesis of RFO, trehalose and cell wall precursors.

Additional file 6: List of DETs related to fatty acid metabolism, carbohydrate and energy metabolism, cell wall loosening and elongation $0,5,10,15$ and $20 \mathrm{dpa}$ in the $\mathrm{fl}$ mutant as compared to their respective stages in WT. Excel file containing the differentially expressed transcripts related to different functional categories based on MIPS and MapMan databases.

Additional file 7: List of primers used for qRT-PCR analysis. Excel file containing the primer sequences used for $\mathrm{QRT}$-PCR to validate microarray data. 


\section{Competing interests}

The authors declare that they have no competing interests.

\section{Authors' contribution}

KVP carried out microarray experiments, analyzed the transcriptome data and drafted the manuscript. KK carried out designing of primers, QRT-PCR analysis and revise the manuscript. GD, MK and MLVP and NJ conducted microarray experiments. SK and DPP involved in the SEM studies. AHP was involved in the SEM and manuscript preparation. TCM, HV and ISK planned and executed experiments to develop stage specific materials and collected stage specific samples for the transcriptome analysis and contributed in the manuscript preparation. VSR, PAK, SL and MKR involved in the designing of the experiments, data analysis, manuscript preparation and discussion. All authors read and approved the manuscript.

\section{Acknowledgments}

This work was supported by funds from the Indian Council of Agricultural Research (ICAR) under the National Agricultural Innovation Project (NAIP), Component-4. Funds from the Department of Biotechnology (DBT), Government of India, International Centre for Genetic Engineering and Biotechnology, New Delhi, India are gratefully acknowledged. KK and DPP acknowledge the DBT and CSIR for the research fellowship, respectively. We thank Dr. B. M. Khadi and Dr N. Gopalakrishnan for providing MCU5 and its near isogenic fuzzless-lintless mutant materials during the initial studies and for their helpful discussions. We also thank Dr. N. K. Singh for the Microarray facility used in the study.

\section{Author details}

${ }^{1}$ National Research Centre on Plant Biotechnology, Pusa Campus, New Delhi 110 012, India. ${ }^{2}$ Plant Transformation Group, International Center for Genetic Engineering and Biotechnology, Aruna Asaf Ali Marg, New Delhi 110067, India. ${ }^{3}$ Agricultural Research Station, Dharwad Farm, University of Agricultural Sciences, Dharwad, India. ${ }^{4}$ Central Institute for Cotton Research, Regional Station, Coimbatore, Tamil Nadu, India.

Received: 9 July 2012 Accepted: 7 November 2012 Published: 14 November 2012

\section{References}

1. Wilkins TA, Arpat AB: The cotton fiber transcriptome. Physiol Plant 2005, 124(3):295-300.

2. Arpat $A B$, Waugh $M$, Sullivan JP, Gonzales M, Frisch D, Main D, Wood T, Leslie A, Wing RA, Wilkins TA: Functional genomics of cell elongation in developing cotton fibers. Plant Mol Biol 2004, 54(6):911-929.

3. Ji SJ, Lu YC, Feng JX, Wei G, Li J, Shi YH, Fu Q, Liu D, Luo JC, Zhu YX: Isolation and analyses of genes preferentially expressed during early cotton fiber development by subtractive PCR and CDNA array. Nucleic Acids Res 2003, 31(10):2534-2543.

4. Wu Y, Machado AC, White RG, Llewellyn DJ, Dennis ES: Expression profiling identifies genes expressed early during lint fibre initiation in cotton. Plant Cell Physiol 2006, 47(1):107-127.

5. Gou JY, Wang $\sqcup$, , Chen SP, Hu WL, Chen XY: Gene expression and metabolite profiles of cotton fiber during cell elongation and secondary cell wall synthesis. Cell Res 2007, 17(5):422-434.

6. Zhao PM, Wang LL, Han LB, Wang J, Yao Y, Wang HY, Du XM, Luo YM, Xia GX: Proteomic identification of differentially expressed proteins in the Ligon lintless mutant of upland cotton (Gossypium hirsutum L.). J Proteome Res 2009, 9(2):1076-1087.

7. Pang CY, Wang H, Pang Y, Xu C, Jiao Y, Qin YM, Western TL, Yu SX, Zhu YX: Comparative proteomics indicates that biosynthesis of pectic precursors is important for cotton fiber and Arabidopsis root hair elongation. $\mathrm{Mol}$ Cell Proteomics 2010, 9(9):2019-2033.

8. Liu K, Han M, Zhang C, Yao L, Sun J, Zhang T: Comparative proteomic analysis reveals the mechanisms governing cotton fiber differentiation and initiation. J Proteomics 2012, 75(3):845-856.

9. Shi YH, Zhu SW, Mao XZ, Feng JX, Qin YM, Zhang L, Cheng J, Wei LP, Wang $Z Y$, Zhu YX: Transcriptome profiling, molecular biological, and physiological studies reveal a major role for ethylene in cotton fiber cell elongation. Plant Cell 2006, 18(3):651-664.
10. Yang SS, Cheung F, Lee JJ, Ha M, Wei NE, Sze SH, Stelly DM, Thaxton P, Triplett B, Town CD, Chen ZJ: Accumulation of genome-specific transcripts, transcription factors and phytohormonal regulators during early stages of fiber cell development in allotetraploid cotton. Plant J 2006, 47(5):761-775.

11. Zhang M, Zheng X, Song S, Zeng Q, Hou L, Li D, Zhao J, Wei Y, Li X, Luo M, Xiao Y, Luo X, Zhang J, Xiang C, Pei Y: Spatiotemporal manipulation of auxin biosynthesis in cotton ovule epidermal cells enhances fiber yield and quality. Nat Biotechnol 2011, 29(5):453-458.

12. Sun Y, Veerabomma S, Abdel-Mageed HA, Fokar M, Asami T, Yoshida S, Allen RD: Brassinosteroid regulates fiber development on cultured cotton ovules. Plant Cell Physiol 2005, 46(8):1384-1391.

13. Luo M, Xiao Y, Li X, Lu X, Deng W, Li D, Hou L, Hu M, Li Y, Pei Y: GhDET2, a steroid 5a-reductase, plays an important role in cotton fiber cell initiation and elongation. Plant J 2007, 51(3):419-430.

14. Machado A, Wu Y, Yang Y, Llewellyn DJ, Dennis ES: The MYB transcription factor GhMYB25 regulates early fibre and trichome development. Plant J 2009, 59(1):52-62.

15. Guan X, Lee JJ, Pang M, Shi X, Stelly DM, Chen ZJ: Activation of Arabidopsis seed hair development by cotton fiber-related genes. PLoS One 2011, 6(7):e21301.

16. Walford SA, Wu Y, Llewellyn DJ, Dennis ES: GhMYB25-like: a key factor in early cotton fibre development. Plant J 2011, 65(5):785-797.

17. Ruan YL, Llewellyn DJ, Furbank RT: Suppression of sucrose synthase gene expression represses cotton fiber cell initiation, elongation, and seed development. Plant Cell 2003, 15(4):952-964.

18. Wang $L$, Ruan $Y L$ : Unraveling mechanisms of cell expansion linking solute transport, metabolism, plasmodesmtal gating and cell wall dynamics. Plant Signal Behav 2010, 5(12):1561-1564.

19. Ruan YL, Xu SM, White R, Furbank RT: Genotypic and developmental evidence for the role of plasmodesmatal regulation in cotton fiber elongation mediated by callose turnover. Plant Physiol 2004, 136(4):4104-4113.

20. Yang YW, Bian SM, Yao Y, Liu JY: Comparative proteomic analysis provides new insights into the fiber elongating process in cotton. $J$ Proteome Res 2008, 7(11):4623-4637.

21. Pang $Y$, Wang $H$, Song WQ, Zhu YX: The cotton ATP synthase $\delta 1$ subunit is required to maintain a higher ATP/ADP ratio that facilitates rapid fibre cell elongation. Plant Biol 2010, 12(6):903-909.

22. Lee J, Burns TH, Light G, Sun Y, Fokar M, Kasukabe Y, Fujisawa K, Maekawa $Y$, Allen RD: Xyloglucan endotransglycosylase/hydrolase genes in cotton and their role in fiber elongation. Planta 2010, 232(5):1191-1205.

23. Liu D, Tu L, Li Y, Wang L, Zhu L, Zhang X: Genes encoding fasciclin-like arabinogalactan proteins are specifically expressed during cotton fiber development. Plant Mol Biol Rep 2008, 26(2):98-113.

24. Harmer SE, Orford SJ, Timmis JN: Characterisation of six alpha-expansin genes in Gossypium hirsutum (upland cotton). Mol Genet Genomics 2002, 268(1):1-9.

25. Li XB, Cai L, Cheng NH, Liu JW: Molecular characterization of the cotton GhTUB1 gene that is preferentially expressed in fiber. Plant Physiol 2002, 130(2):666-674.

26. Li XB, Fan XP, Wang XL, Cai L, Yang WC: The cotton ACTIN1 gene is functionally expressed in fibers and participates in fiber elongation. Plant Cell 2005, 17(3):859-875.

27. Wang J, Wang HY, Zhao PM, Han LB, Jiao GL, Zheng YY, Huang SJ, Xia GX: Overexpression of a profilin (GhPFN2) promotes the progression of developmental phases in cotton fibers. Plant Cell Physiol 2010, 51(8):1276-1290.

28. Wang QQ, Liu F, Chen XS, Ma XJ, Zeng HQ, Yang ZM: Transcriptome profiling of early developing cotton fiber by deep-sequencing reveals significantly differential expression of genes in a fuzzless/lintless mutant. Genomics 2010, 96(6):369-376.

29. Peter SD, Muralidharan V, Bhat MV: Fuzzless-lintless mutant of MCU 5 cotton. Cotton Dev 1984, 14:43.

30. Nadarajan N, Rangagasamy SRS: Breeding value of a fuzzless-lintless mutant in Gossypium hirsutum L. Indian J Genet 1997, 57(3):233-237.

31. Oh JE, Kwon Y, Kim JH, Noh H, Hong SW, Lee H: A dual role for MYB60 in stomatal regulation and root growth of Arabidopsis thaliana under drought stress. Plant Mol Biol 2011, 77(1-2):91-103.

32. Suzuki N, Rizhsky L, Liang H, Shuman J, Shulaev V, Mittler R: Enhanced tolerance to environmental stress in transgenic plants expressing the 
transcriptional coactivator multiprotein bridging factor 1c. Plant Physiol 2005, 139(3):1313-1322.

33. Gao P, Zhao PM, Wang J, Wang HY, Wu XM, Xia GX: Identification of genes preferentially expressed in cotton fibers: A possible role of calcium signaling in cotton fiber elongation. Plant Sci 2007, 173(1):61-69.

34. Huang QS, Wang HY, Gao P, Wang GY, Xia GX: Cloning and characterization of a calcium dependent protein kinase gene associated with cotton fiber development. Plant Cell Rep 2008 27(12):1869-1875.

35. George L, Romanowsky SM, Harper JF, Sharrock RA: The ACA10 $\mathrm{Ca}^{2+}$-ATPase regulates adult vegetative development and inflorescence architecture in Arabidopsis. Plant Physiol 2008, 146(2):716-728.

36. Blakeslee JJ, Bandyopadhyay A, Lee OR, Mravec J, Titapiwatanakun B, Sauer M, Makam SN, Cheng Y, Bouchard R, Adamec J, Geisler M, Nagashima A, Sakai T, Martinoia E, Friml J, Peer WA, Murphy AS: Interactions among PIN-FORMED and P-Glycoprotein Auxin Transporters in Arabidopsis. Plant Cell 2007, 19(1):131-147.

37. Molnar G, Bancos S, Nagy F, Szekeres M: Characterisation of BRH1, a brassinosteroid-responsive RING-H2 gene from Arabidopsis thaliana. Planta 2002, 215(1):127-133.

38. Brill E, van Thournout M, White RG, Llewellyn D, Campbell PM, Engelen S, Ruan YL, Arioli T, Furbank RT: A novel isoform of sucrose synthase is targeted to the cell wall during secondary cell wall synthesis in cotton fiber. Plant Physiol 2011, 157(1):40-54

39. Yeo ET, Kwon HB, Han SE, Lee JT, Ryu JC, Byu MO: Genetic engineering of drought resistant potato plants by introduction of the trehalose-6phosphate synthase (TPS1) gene from Saccharomyces cerevisiae. Mol Cells 2000, 10(3):263-268.

40. Padmalatha KV, Dhandapani G, Kanakachari M, Kumar S, Dass A, Patil DP Rajamani V, Kumar K, Pathak R, Rawat B, Leelavathi S, Reddy PS, Jain N, Powar KN, Hiremath V, Katageri IS, Reddy MK, Solanke AU, Reddy VS, Kumar PA: Genome-wide transcriptomic analysis of cotton under drought stress reveal significant down-regulation of genes and pathways involved in fibre elongation and up-regulation of defense responsive genes. Plant Mol Biol 2012, 78(3):223-246.

41. Juszczuk IM, Szal B, Rychter AM: Oxidation-reduction and reactive oxygen species homeostasis in mutant plants with respiratory chain complex I dysfunction. Plant Cell Environ 2012, 35(2):296-307.

42. Qin YM, Pujol FM, Shi YH, Feng JX, Liu YM, Kastaniotis AJ, Hiltunen JK, Zhu $Y X$ : Cloning and functional characterization of two CDNAs encoding NADPH-dependent 3-ketoacyl-CoA reductased from developing cotton fibers. Cell Res 2005, 15(6):465-473.

43. Qin YM, Pujol FM, Hu CY, Feng JX, Kastaniotis AJ, Hiltunen JK, Zhu YX: Genetic and biochemical studies in yeast reveal that the cotton fibrespecific GhCER6 gene functions in fatty acid elongation. J Exp Bot 2007, 58(3):473-481

44. Flugge UI, Hausler RE, Ludewig F, Gierth M: The role of transporters in supplying energy to plant plastids. J Exp Bot 2011, 62(7):2381-2392.

45. Leung KC, Li HY, Mishra G, Chye ML: ACBP4 and ACBP5, novel Arabidopsis acyl-CoA-binding proteins with kelch motifs that bind oleoyl-CoA. Plant Mol Biol 2004, 55(2):297-309.

46. Anthony RG, Henriques R, Helfer A, Meszaros T, Rios G, Testerink C, Munnik $T$, Deak M, Koncz C, Bogre L: A protein kinase target of a PDK1 signalling pathway is involved in root hair growth in Arabidopsis. EMBO J 2004, 23(3):572-581.

47. Bellevik S, Zhang J, Meijer J: Brassica napus soluble epoxide hydrolase (BNSEH1). Eur J Biochem 2002, 269(21):5295-5302.

48. Bird DA: The role of $A B C$ transporters in cuticular lipid secretion. Plant SCi 2008, 174(6):563-569.

49. Yeats TH, Rose JKC: The biochemistry and biology of extracellular plant lipid-transfer proteins (LTPs). Protein Sci 2008, 17(2):191-198.

50. Zhu YQ, Xu KX, Luo B, Wang JW, Chen XY: An ATP-binding cassette transporter GhWBC1 from elongating cotton fibers. Plant Physiol 2003, 133(2):580-588.

51. Luo $B$, Xue $X Y$, Hu WL, Wang $L$, Chen $X Y$ : An $A B C$ transporter gene of Arabidopsis thaliana, AtWBC11, is involved in cuticle development and prevention of organ fusion. Plant Cell Physiol 2007, 48(12):1790-1802

52. DeBono A, Yeats TH, Rose JKC, Bird D, Jetter R, Kunst L, Samuels L: Arabidopsis LTPG is a glycosylphosphatidylinositol-anchored lipid transfer protein required for export of lipids to the plant surface. Plant Cell 2009, 21(4):1230-1238.
53. Liu D, Tu L, Wang L, Li Y, Zhu L, Zhang $X$ : Characterization and expression of plasma and tonoplast membrane aquaporins in elongating cotton fibers. Plant Cell Rep 2008, 27(8):1385-1394.

54. Park W, Scheffler BE, Bauer PJ, Campbell BT: Identification of the family of aquaporin genes and their expression in upland cotton (Gossypium hirsutum L.). BMC Plant Biol 2010, 10:142.

55. Jou Y, Chiang CP, Jauh GY, Yen HE: Functional characterization of ice plant SKD1, an AAA-type ATPase associated with the endoplasmic reticulum-Golgi network, and its role in adaptation to salt stress. Plant Physiol 2006, 141(1):135-146.

56. Wang H, Guo Y, Lv F, Zhu H, Wu S, Jiang Y, Li F, Zhou B, Guo W, Zhang T: The essential role of GhPEL gene, encoding a pectate lyase, in cell wall loosening by depolymerization of the de-esterified pectin during fiber elongation in cotton. Plant Mol Biol 2010, 72(4-5):397-406.

57. Bischoff V, Nita S, Neumetzler L, Schindelasch D, Urbain A, Eshed R, Persson S, Delmer D, Scheible WR: TRICHOME BIREFRINGENCE and its homolog AT5G01360 encode plant-specific DUF231 proteins required for cellulose biosynthesis in Arabidopsis. Plant Physiol 2010, 153(2):590-602.

58. Mir IH, Channa A: A Scanning Electron Microscopic Examination of the Intestinal Tract of the Snow Trout, Schizothorax curvifrons Heckel. J Fish Aquat Sci 2010, 5(5):386-393.

59. Benjamini Y, Hochberg Y: On the adaptive control of the false discovery rate in multiple testing with independent statistics. J Educ Behav Stat 2000, 25(1):60-83.

60. Eisen MB, Spellman PT, Brown PO, Botstein D: Cluster analysis and display of genome-wide expression patterns. Proc Natl Acad Sci 1998, 95:14863-14868.

61. Artico S, Nardeli SM, Brilhante O, Grossi-de-Sa MF, Alves-Ferreira M: Identification and evaluation of new reference genes in Gossypium hirsutum for accurate normalization of real-time quantitative RT-PCR data. BMC Plant Biol 2010, 10:49.

doi:10.1186/1471-2164-13-624

Cite this article as: Padmalatha et al:: Functional genomics of fuzzlesslintless mutant of Gossypium hirsutum L. Cv. MCU5 reveal key genes and pathways involved in cotton fibre initiation and elongation. BMC Genomics 2012 13:624.

\section{Submit your next manuscript to BioMed Central and take full advantage of:}

- Convenient online submission

- Thorough peer review

- No space constraints or color figure charges

- Immediate publication on acceptance

- Inclusion in PubMed, CAS, Scopus and Google Scholar

- Research which is freely available for redistribution 